\title{
Odd Triperfect Numbers Are Divisible By Eleven Distinct Prime Factors
}

\author{
By Masao Kishore
}

Abstract. We prove that an odd triperfect number has at least eleven distinct prime factors.

1. Introduction. A positive number $N$ is called a triperfect number if $\sigma(N)=3 N$ where $\sigma(N)$ is the sum of the positive divisors of $N$. Six even triperfect numbers are known:

$$
\begin{aligned}
& 2^{14} \cdot 5 \cdot 7 \cdot 19 \cdot 31 \cdot 151 \\
& 2^{13} \cdot 3 \cdot 11 \cdot 43 \cdot 127 \\
& 2^{9} \cdot 3 \cdot 11 \cdot 31 \\
& 2^{8} \cdot 5 \cdot 7 \cdot 19 \cdot 37 \cdot 73 \\
& 2^{5} \cdot 3 \cdot 7 \\
& 2^{3} \cdot 3 \cdot 5
\end{aligned}
$$

However, the existence of an odd triperfect (OT) number is an open question. McDaniel [4] and Cohen [2] proved that an OT number has at least nine distinct prime factors; the author proved that it has at least ten prime factors [3], and Beck and Najar [1] showed that it exceeds $10^{50}$.

In this paper we prove

THEOREM. If $N$ is $O T, N$ has at least eleven distinct prime factors.

2. Proof of Theorem. Throughout this paper we let

$$
N=\prod_{i=1}^{10} p_{i}^{a_{i}},
$$

where $p_{i}$ 's are odd primes, $p_{1}<\cdots<p_{10}$ and $a_{i}$ 's are positive integers. We call $p_{i}^{a_{i}}$ a component of $N$ and write $p_{i}^{a_{i}} \| N$.

The following lemmas are easy to prove:

LEMMA 1. If $N$ is $O T, a_{i}$ 's are even for $1 \leqslant i \leqslant 10$.

LEMMA 2. If $N$ is $O T$ and $q$ is a prime factor of $\sigma\left(p_{i}^{a_{i}}\right)$ for some $i$, then $q=3$ or $q=p_{j}$ for some $j, 1 \leqslant j \leqslant 10$.

The following lemmas are stated in [5].

Received April 4, 1983; revised June 5, 1984.

1980 Mathematics Subject Classification. Primary 10A20. 
Lemma 3. Suppose $q$ is a prime, $q \geqslant 2$ and $a \geqslant 1$. Then $\sigma\left(q^{a}\right)$ has a prime factor $p$ such that $a+1$ is the order of $q$ modulo $p$ except for $q=2$ and $a=5$ and for $q=a$ Mersenne prime and $a=1$. In particular $a+1 \mid p-1$.

Lemma 4. Suppose $p$ is a Fermat prime (3,5,17, etc.), $q$ is an odd prime and a is even. If $p^{b} \mid \sigma\left(q^{a}\right)$, then $q \equiv 1(p), p^{b} \mid a+1$, and $\sigma\left(q^{a}\right)$ has $b$ distinct prime factors congruent to 1 modulo $p$.

LEMMA 5. If $N$ is $O T, 17+N$.

Proof. Suppose $N$ is OT. Since the three smallest primes $\equiv 1$ (17) are 103, 137, and 239 and

$$
\frac{3}{2} \frac{5}{4} \frac{7}{6} \frac{11}{10} \frac{13}{12} \frac{17}{16} \frac{19}{18} \frac{103}{102} \frac{137}{136} \frac{239}{238}<3,
$$

$N$ has at most two primes $\equiv 1$ (17). Suppose $p^{a}$ and $q^{b}$ are components of $N$ and $p \equiv q \equiv 1$ (17). If $17^{c} \mid N$ and $c \geqslant 4$, then $17^{2} \mid \sigma\left(p^{a}\right)$ or $17^{2} \mid \sigma\left(q^{b}\right)$, and, by Lemma $4, N$ would have two more primes $\equiv 1(17)$, a contradiction. Hence $17^{4}+N$. Suppose $17^{2} \| N$. Then $N$ has a component $307^{d}$ because $\sigma\left(17^{2}\right)=307$. Then $17+\sigma\left(307^{d}\right)$ because $16661 \cdot 36857\left|\sigma\left(307^{16}\right), \sigma\left(307^{16}\right)\right| \sigma\left(307^{d}\right)$ and

$$
\frac{3}{2} \frac{5}{4} \frac{7}{6} \frac{11}{10} \frac{13}{12} \frac{17}{16} \frac{19}{18} \frac{307}{306} \frac{16661}{16660} \frac{36857}{36856}<3 .
$$

Hence $N$ has another component $p^{b}$ such that $17^{2} \mid \sigma\left(p^{b}\right)$. Then we get a contradiction again. Hence $17+N$. Q.E.D.

The proof of the following lemma is easy.

LEMMA 6. If $N$ is $O T, p_{9} \leqslant 283$.

LEMMA 7. If $N$ is $O T$ and $5^{a} \| N$, then $a=2,5^{2} \mid \sigma\left(P_{10}^{a_{10}}\right)$ and $p_{10} \geqslant 311$.

Proof. Suppose $N$ is OT, $p^{b}$ is a component of $N$ and $5 \mid \sigma\left(p^{b}\right)$. By Lemma $4, p \equiv$ $1(5), 5 \mid b+1$ and $\sigma\left(p^{4}\right) \mid \sigma\left(p^{b}\right)$. If $61 \leqslant p \leqslant 281$, then $\sigma\left(p^{4}\right)$ has a prime factor $q$ such that

$$
\frac{3}{2} \frac{5}{4} \frac{7}{6} \frac{11}{10} \frac{13}{12} \frac{19}{18} \frac{23}{22} \frac{29}{28} \frac{p}{p-1} \frac{q}{q-1}<3, \text { or }
$$

$\sigma\left(p^{4}\right)$ has prime factors $q$ and $r$ such that

$$
\frac{3}{2} \frac{5}{4} \frac{7}{6} \frac{11}{10} \frac{13}{12} \frac{19}{18} \frac{23}{22} \frac{p}{p-1} \frac{q}{q-1} \frac{r}{r-1}<3 .
$$

Hence $p=11,31$ or 41 or $p \geqslant 311$.

Suppose $p=11,31$ or 41 . If $5^{2}\left|\sigma\left(p^{b}\right), 5^{2}\right| b+1$ by Lemma 4 . Then $\sigma\left(p^{24}\right) \mid \sigma\left(p^{b}\right)$ and $\sigma\left(p^{24}\right)$ has two distinct prime factors $>283$, contradicting Lemma 6. Hence $5^{2}+\sigma\left(p^{b}\right)$. Since $3221\left|\sigma\left(11^{4}\right), 17351\right| \sigma\left(31^{4}\right)$ and $579281 \mid \sigma\left(41^{4}\right)$, $5^{2}+\sigma\left(\Pi_{i=1}^{9} p_{i}^{a_{i}}\right)$ and $p_{10}=3221,17351$ or 579281 and $5 \mid \sigma\left(p_{10}^{a_{10}}\right)$. However, $\sigma\left(p_{10}^{4}\right)$ has a prime factor $>283$, contradicting Lemma 6 . Hence $p \geqslant 311$ and $5^{a} \mid \sigma\left(p^{b}\right)$.

If $a \geqslant 4$, then by Lemma $4, N$ would have four more primes $\equiv 1$ (5), which is a contradiction because

$$
\frac{3}{2} \frac{5}{4} \frac{7}{6} \frac{11}{10} \frac{13}{12} \frac{19}{18} \frac{31}{30} \frac{41}{40} \frac{61}{60} \frac{311}{310}<3
$$

Hence $a=2$. Q.E.D. 
LEMMA 8. If $N$ is $O T, p_{9} \leqslant 71$.

Proof. By Lemma 6, $31=\sigma\left(5^{2}\right) \mid N$. Since

$$
\frac{3}{2} \frac{\sigma\left(5^{2}\right)}{5^{2}} \frac{7}{6} \frac{11}{10} \frac{13}{12} \frac{19}{18} \frac{23}{22} \frac{31}{30} \frac{73}{72} \frac{311}{310}<3
$$

$p_{9} \leqslant 71$. Q.E.D.

Proof of Theorem. If $N$ is OT, then by Lemmas 4 and $7,5^{2}\left|\sigma\left(p_{10}^{a_{10}}\right), 5^{2}\right| a_{10}+1$ and $\sigma\left(p_{10}^{24}\right) \mid\left(p_{10}^{a_{0}}\right)$. By Lemma 3, $\sigma\left(p_{10}^{24}\right)$ has a prime factor $q$ such that $25 \mid q-1$. Hence $q=25 b+1$ for some $b$. Since $q$ is a prime, $b \neq 1$ or 2 . Then $q>71$ and $q \neq p_{10}$, contradicting Lemma 8. Q.E.D.

Department of Mathematics

East Carolina University

Greenville, North Carolina 27834

1. W. E. Beck \& R. M. NaJaR, "A lower bound for odd triperfects," Math. Comp., v. 38, 1982, pp. 249-251.

2. G. L. CoHEN, "On odd perfect numbers II, Multiperfect numbers and quasiperfect numbers," $J$. Austral. Math. Soc., v. 29, 1980, pp. 369-384.

3. M. KishORE, "Odd triperfect numbers," Math. Comp., v. 42, 1984, pp. 231-233.

4. W. MCDANIEL, "On odd multiply perfect numbers," Boll. Un. Mat. Ital. (4), v. 3, 1970, pp. 185-190.

5. C. Pomerance, "Odd perfect numbers are divisible by at least seven distinct primes," Acta Arith., v. $25,1973 / 1974$, pp. $265-300$. 